\title{
Über die Entstehungszeit der „Himmelfahrt Moses“.
}

\author{
Von Gustav Hölscher in Halle a. S.
}

\author{
I.
}

In den Monumenta sacra et profana vol. I. fasc. I, pp. 55-64 veröffentlichte Ceriani 1861 nach dem einzigen vorhandenen Mailänder Palimpseste jenes bedeutende Bruchstück einer ursprünglich jüdischen Moseapokalypse, die seitdem als Assumptio Mosis bekannt ist. In dieser Schrift weissagt Moses vor seinem Abscheiden dem Josua die künftigen Schicksale Israels bis zum Ende der Tage. Von einer „Himmelfahrt" Moses ist in dem vorliegenden Bruchstücke nur in der offenkundigen Glosse 10,12 (receptione) die Rede; da die ursprüngliche Schrift von einer solchen nicht erzählt zu haben scheint, sondern nach biblischer Vorstellung den Gesetzgeber eines natürlichen Todes sterben läßt $(1,15 ; 3,13 ; 10,12.14)$, so wäre sie richtiger als „Testament Moses" $\mathrm{zu}$ bezeichnen. Der allein erhaltene lateinische Text ist anerkanntermaßen die Übersetzung einer griechischen Vörlage, die ihrerseits wieder auf einen semitischen, und zwar anscheinend hebräischen Grundtext zurückgeht.

Über die Entstehungszeit dieser Apokalypse ist bald nach ihrer Wiederentdeckung lebhaft gestritten worden. Daß sie nach der 34 jährigen Regierung Herodes des Älteren verfaßt sei, ergab sich aus 6, 6. Alles übrige dagegen blieb lange umstritten. Während die einen das Buch bald hinter den Tod des Herodes $\left(4 \mathrm{v}\right.$. Chr.) ${ }^{1}$ oder hinter die Absetzung seines Sohnes Archelaos (6 n. Chr.), ${ }^{2}$ andere in die Jahrzehnte vor ${ }^{3}$ oder nach $70^{4}$ setzten, rückte eine Minderzahl von Forschern

1 So Ewald. (1862), Wieseler (1868), Dillmann (1883), Schürer (1886), Deane (1891), Faye (1892), Clemen (1899), Burkitt (1900), Bousset (1903).

2 So Drummond (1877), Thomson (1891), Charles (1897).

3 Hilgenfeld (1867): 44-45; Fritzsche (1871) und Lucius (1881): 50-60; Schmidt und Merx (1868): 54-64; Baldensperger (1888): 50-70; Briggs (1895): vor 70.

4 Langen (1866), Keim (1867ff.) und Rosenthal (1885): bald nach 70; Hausrath $(1873 \mathrm{ff}):. 81-96$. 
es bis ins zweite Jahrhundert $\mathrm{n}$. Chr., bis in die Zeit Hadrians hinab. ${ }^{1}$ Allmählich ist der Streit fast verstummt; man hat sich daran gewöhnt, das Buch in die Zeit der Herodessöhne zu' setzen. Die Gründe, mit denen man diese Datierung stützt, erscheinen den Auslegern offenbar ganz und gar durchschlagend, während die Schwierigkeiten, von denen sie meines. Erachtens schwer bedrückt ist, nicht empfunden werden. Die folgende Untersuchung nimmt die Frage der Datierung von neuem auf. Ihr Ergebnis nähert sich jener Ansicht, die schon von Forschern, wie Volkmar, Colani u. a., ausgesprochen worden ist. So wenig einwandfrei die von ihnen gegebene Begründung im einzelnen sein mag, ihren Versuch, das Buch aus der hadrianischen Verfolgungszeit zu begreifen, halte ich doch noch immer für höchst beachtenswert; und daß er besser, als es geschehen ist, begründet werden kann, hoffe ich im folgenden $z u$ zeigen.

1.

Als Stütze der heute herrschenden Datierung gilt vor allem der Satz 6,7. Bis dahin hat der Verfasser unsers Buches die Geschichte Israels vom Tode Moses bis ins zweite vorchristliche Jahrhundert (1-5), sodann das Auftreten der hasmonäischen Priesterkönige $(6,1)$ und schließlich die grausame Herrschaft Herodes des Älteren $(6,2-6)$ nach apokalyptischer Art weissagend dargestellt. Nun fährt er 6,7 fort: Et [p]roducet natos, [qui succ]edentes sibi breviora tempora do(mi)narent. Bekanntlich trifft die hier gegebene Zeitbestimmung für die Söhne des Herodes nur teilweise zu. Denn nur Archelaos (4 vor-6 n. Chr.) regierte kürzere Zeit als sein Vater, während Antipas (4 vor-39 n. Chr.) und Philippos (4 vor-34 n. Chr.) um einige Jahre länger auf dem Throne saßen. Dieser Umstand pflegt

1 Volkmar (1867), Colani (1868) und Loeb (1888): 137-138; Philippi (1868): zweites Jahrhundert.

2 Wenn Volkmar (Mose Prophetie und Himmelfahrt 1867) die Schrift in das Jahr 137-138 n. Chr. setzte und den vielumstrittenen TAXO 9, 1 auf Rabbi Akiba deutete, so huldigte er auch hier seiner bekannten und oft bedenklichen Vorliebe für die hadrianische Zeit. I $\mathrm{hm}$ folgte in der Frage der Datierung Colani (L'assomption de Moïse in der Revue de théologie IV 1868 pp. 65-94), jedoch bezog er den TAXO auf Rabbi Jehuda ben Baba, welcher, ehe er im Jahre 137 von den Römern erschlagen wurde, die sieben letzten Schüler Akibas in einer Schlucht bei Uscha ordiniert haben soll. Bei Philippi (Das Buch Henoch 1868 S. 166-191) ist die Datierung ins zweite Jahrhundert nicht unabhängig von dem Wunsche, das Buch einem Christen zuzuschreiben. Auf Josua ben Chananja deutete den TAXO Isidor Loeb (Le taxo de l'assomption de Moîse, Extrait de l'Univers israélite, 35e année no. 2, vgl. dazu den Bericht von Erneste Renan im Journal asiatique, septième série t. XVI 1880 p. 45). 
den Auslegern zu genügen, um ohne weiteres zu schließen, daß der Verfasser des Buches den Tod des Philippos im Jahre $34 \mathrm{n}$. Chr. noch nicht erlebt haben könne. Aber schon Forscher wie Reuß, Rosenthal und Baldensperger haben die Berechtigung einer solchen Schlußfolgerung in begründeten Zweifel gezogen. Mit Recht bemerkt Reuß, daß nati (בנים) auch allgemeiner die Nachkommen des Herodes bezeichnen könne, und daß der Verfasser an die Tetrarchen Philippos und Antipas vielleicht gar nicht gedacht, sondern nur Archelaos und etwa noch Agrippa I (41-44 n. Chr.) im Auge gehabt habe, „die allein für einen Jerusalemer Interesse hatten ". Das wäre vollends leicht erklärlich, wenn der Verfasser nicht zu Anfang des ersten Jahrhunderts, sondern erst hundert Jahre später geschrieben hätte. Auf jeden Fall ist soviel klar, daß der Satz 6, 7 an und für sich eine Abfassung des Buches zur Zeit der Herodessöhne schlechterdings nicht beweist. Ja, er spricht eher gegen als für sie; denn es sieht nicht darnach aus, als ob der einfache Relativsatz: [qui] . . . breviora tempora do(mi)narent, dessen Zeitangabe wie ein gewöhnliches apokalyptisches vaticinium ex eventu klingt, den Sinn einer schweren zeitgemäßen Drohung gegen Antipas und Philippos haben sollte, wie die übliche Auslegung der Stelle es nötig machen würde. Es kommt noch ein weiteres hinzu: da die Fortsetzung 6, 8-9 nach allgemeiner Auffassung nicht Zukünftiges, sondern schon Erlebtes beschreibt, so wird auch der vorhergehende Satz 6,7 schwerlich reine Drohweissagung sein. Schon der Satz 6,7 scheint also nahezulegen, daß der Verfasser nicht bald nach dem Tode des Herodes, sondern beträchtlich später schrieb.

Der Text fährt in 6,8-9 fort: in partes eorum cohortes venient et occidentis rex potens qui expugnabit eos et ducet captivos et partem aedis ipsorum igni incendet (et) aliquos crucifiget circa coloniam eorum. Man kann das in die Grundsprache etwa so zurückübersetzen: בגבולם

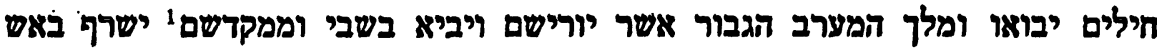
. Fast allgemein deutet man diese Stelle auf P. Quinctilius Varus, der nach dem Tode des Herodes im Jahre 4 v. Chr. zur Unterdrückung eines Aufstandes nach Jerusalem kam. Die

1 Das einzige sonst vorhandene hebräische (aramäische) Wort für "Teil " wäre

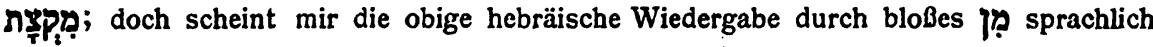
wahrscheinlicher, wie dies auch 4, 7 vorliegen könnte: tunc ascendent aliquae partes tribuum = יעלו מן־השבטים IX. Sonst gibt der Ubersetzer durch pars auch hebräisches קצrenze) wieder, wie 3,13: usque nos duci captivos in partem orientis

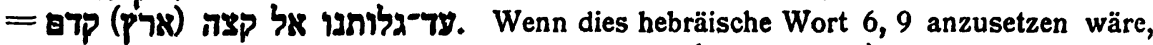

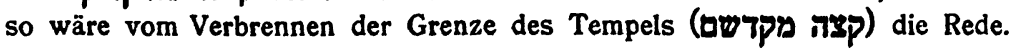


Kenntnis der damaligen Ereignisse verdanken wir der zuverlässigen Erzählung bei Josephus (bell. II 39-79, ant. XVII 250-298), welche auf die zeitgenössische Darstellung des Nikolàòs von Damaskos zurückgehen dürfte. Dort wird berichtet, wie nach einer erstmaligen Anwesenheit des Varus in Jerusalem die Soldaten des Prokurators Sabinus das Dach der den Tempelplatz umgebenden Säulenhallen anzündeten, in das Heiligtum eindrangen und den Tempelschatz ausraubten; wie darnach der Aufstand im ganzen Lande um sich griff, Varus von neuem mit zwei Legionen anrückte, Galiläa säuberte, Sepphoris in Brand steckte und dessen Bewohner als Sklaven verkaufte, dann über Samaria nach Jerusalem zog und ohne Widerstand in die Hauptstadt einrückte, um von dort aus die im Lande zerstreuten Scharen der Empörer einzufangen und zweitausend von ihnen zu kreuzigen, während er der Masse des Volkes Verzeihung gewährte. Dies die Tatsachen, wie sie Josephus überliefert. $\mathrm{DaB}$ sie sich mit den Angaben unseres Textes 6, 8-9 nicht decken, ist offensichtlich. Schon die Bezeichnung occidentis rex potens paßt nicht auf einen römischen Legaten oder Prokurator, sondern offenbar nur auf einen Kaiser. Sie erinnert deutlich an die Ausdrucksweise des Danielbuchs, mit dem sich unsere Schrift auch sonst gelegentlich berührt. Dan $11-12$ bezeichnet die Ptolemäer

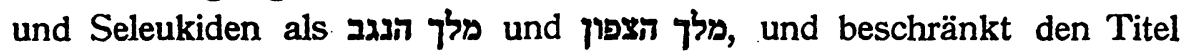
מלך durchweg auf wirkliche Könige, während z. B. der römische Konsul und Feldherr Lucius Scipio, der Sieger von Magnesia, קצין (Dan 11, 18), Seleukos als Feldherr des Ptolemaios I. שר (Dan 11,5) und der Steuereinnehmer Heliodoros נוגש (Dan 11, 20) heißt. Mit könnte also höchstens, wie Volkmar es vorschlug, Augustus bezeichnet sein, aber dieser ist im Jahre $4 \mathrm{v}$. Chr. nicht nach Syrien gekommen.

Auch die Folge der Ereignisse, wie sie 6, 8-9 beschrieben werden, deckt sich nicht mit dem Berichte über den Varuskrieg bei Josephus, was um so auffälliger sein müßte, wenn unser Apokalyptiker ein Zeitgenosse jener Ereignisse gewesen wäre. ${ }^{\mathrm{Er}}$ läßt den „mächtigen König des Westens " das Land erobern, Gefangene wegführen, einen Teil des Tempels verbrennen und einige rings um die Stadt kreuzigen. Varus dagegen kam aus Antiochia nach Palästina, nachdem die Soldaten des Sabinus das Dach der Tempelhallen angezündet hatten; von Gefangenen, die Varus als Sklaven verkaufen lieB, wird nur nach der Einnahme von Sepphoris erzählt - an die der Apokalyptiker schwerlich gedacht hat, - während von einer Wegführung der Jerusalemer bei Josephus nicht die Rede ist.

Die Beziehung der Stelle 6,8-9 auf die Ereignisse des Varus- 
krieges ist mithin keineswegs so einfach, wie die übliche Auslegung voraussetzt. Wenn dagegen 6, 7 mit Recht auf Nachkommen des $\mathrm{He}-$ rodes, wie Archelaos und Agrippa I. gedeutet worden ist, so bietet sich die Beziehung von 6, 8-9 auf die Ereignisse des Jahres 70 von selber. Der "mächtige König des Westens" ist dann kein anderer als Vespasian oder Titus. Titus war es, der nach der Einnahme der Stadt alle, die noch nicht dem Hunger oder dem Schwerte erlegen waren, teils hinrichten ließ, teils in die ägyptischen Bergwerke verschickte, zu Gladiatorenkämpfen bestimmte oder als Sklaven verkaufen ließ (bell. VI 414-419; VII 21-36). Noch die spätere jüdische Überlieferung redet von der damaligen Wegführung Israels nach Rom (Midrasch über die zehn Exile, vgl. Aug. Wünsche: Aus Israels Lehrhallen II. Band, 1908, S. 93). Bei der Eroberung der Stadt durch Titus geschah es, daß zahllose Juden vor den Toren der Stadt gekreuzigt (bell. V $449 \mathrm{ff}$., vita 420) und das Tempelgebäude ein Raub der Flammen ward (bell. VI $220-266)$.

Es ist nicht wahrscheinlich, daß jener gelegentliche Brand des Tempelhallendachs im Jahre $4 \mathrm{v}$. Chr. dem Apokalyptiker so bemerkenswert erschienen wäre, daß er ihn als Vorzeichen des nahenden Weltendes hätte ansehen sollen. Wie anders der Tempelbrand des Jahres 70, welcher das Ende des Kultes auf Zion bedeutete! Daß auch der Ausdruck partem aedis ipsorum diese Deutung nicht hindert, wird unten zur Sprache kommen.

Zunächst müssen wir der Darstellung weiter folgen. Der Verfasser fährt in 7,1 fort: ex quo facto finientur tempora. Hier soll nach Ansicht der Ausleger die eigentliche Zukunftsschilderung beginnen. So erklärt Burkitt (in Hastings Dictionary of the Bible III 449): „Hier endet die Geschichte und die Weissagungen beginnen: zuerst sollen Regenten kommen, welche heuchlerisch, schwelgend, tyrannisch, gottlos, prahlerisch, hochmütig sein werden . . . (7); darauf wird eine andere Zornesheimsuchung über sie kommeî, und "der König der Könige der Erde« wird die, die ihre Beschneidung bekennen, kreuzigen und ihre Frauen den Heiden geben und sie zwingen, unreine Götzenbilder zu tragen und zu lästern (8). Dann wird ein Mann vom Stamme Levi auftreten, dessen Name TAXO sein wird usw." Burkitt verlegt demnach den gesamten Inhalt von c. 7-10 folgerichtig in die Zukunft. Andere Ausleger sind nicht ebenso streng in ihrer Folgerung, sondern verstehen das Auftreten der Gottlosen in 7,3-9 noch als Gegenwart des Verfassers. Das ist offenbar exegetisch richtig empfunden. Dann beginnt aber die Zukunftsschilderung nicht mit 7,1, und der Satz finientur tempora 7,1 
bezeichnet dann nicht den unmittelbaren Eintritt des Weltendes, sondern den Beginn der letzten Zeiten vor dem Ende.

Wann dies Ende eintreten wird, beantwortete die Zahlenrechnung 7, 1-2. Eben sie ist das eigentliche Geheimnis, welches der Apokalyptiker seinen Lesern entschleiern will. Leider ist ihr Text so lückenhaft erhalten, daß die neueren Ausleger, soweit mir bekannt ist, durchweg auf eine Erklärung verzichten. Man kann das begreifen, da jede Herstellung des Textes in der Tat nur als ein Versuch gelten kann; aber es ist bedenklich, auch die deutlichen Aussagen der Stelle, wie es meist üblich geworden ist, einfach beiseite $\mathrm{zu}$ schieben. Soviel ist nämlich klar, daß der Verfasser von jener 6, 8-9 erwähnten Eroberung des Landes durch den König des Westens und dem Tempelbrande bis zum Eintritt des Endes einen längeren, in mehrere Abschnitte sich gliedernden Zeitraum rechnete. Er redet von „vier Stunden", die kommen sollen, und bringt dann eine ziemlich verwickelte Rechnung mit mancherlei Zahlen. Zweierlei ist dabei ersichtlich: erstens, daß der berechnete Zeitraum nicht ganz kurz ist, und zweitens, daß ein Teil jener Zeitabschnitte für den Verfasser schon vergangen ist; denn sonst hätte er den Zeitraum bis zum Eintritt des Endes weit einfacher bestimmen können. Das ist denn auch die Meinung aller derer, die je einen ernstlichen Versuch zur Textherstellung in 7,1-2 gemacht haben, so verschieden auch die Verbesserungsvorschläge im einzelnen sind. Sie alle sind $\mathrm{zu}$ dem Schlusse gekommen, daß die Schrift eine längere Zeit nach den in 6, 8-9 angedeuteten Ereignissen geschrieben sein müsse, ${ }^{1}$ während umgekehrt diejenigen, die die Schrift bald nach dem Tode des Herodes geschrieben sein lassen, bezeichnenderweise auf jede Berücksichtigung der Zahlenrechnung in 7,1-2 zu verzichten pflegen. Selbst wenn man also 6,8-9 auf den Varuskrieg beziehen wollte, wie es auch Hilgenfeld, Schmidt, Merx und Volkmar tun, könnte die Schrift erst geraume Zeit nach demselben verfaßt sein; bezieht man dagegen 6, 8-9 richtiger auf den Krieg des Titus, so schrieb der Verfasser eine entsprechende Zeit nach diesem Ereignis.

1 Hilgenfeld und Schmidt-Merx verstehen die „vier Stunden ${ }^{*}$ von den Regierungen der Kaiser Augustus, Tiberius, Caius und Claudius; Volkmar dagegen deutet sie auf vier Perioden von Regierungen: a) Augustus bis Nero, b) Galba, Otho, Vitellius, c) Vespasian, Titus, Domitian, d) Nerva, Trajan, Hadrian. - Ganz willkürlich ist die Berechnung Wieselers, der ex quo facto 7,1 auf die Eroberung Jerusalems durch Pompeius $63 \mathrm{v}$. Chr. bezieht, die vorher überhaupt nirgends auch nur angedeutet worden ist.

Zeitschr. f. d. neutest. Wiss. 17. Jahrg. 1916. 
Daraus ergibt sich das weitere. Die Gottlosen, die 7, 8-9 beschrieben werden, sind Leute der Zeit nach 70. Es leuchtet ein, daß es keine Gestalten apokalyptischer Phantasie sind. Ihr Bild ist recht nach dem Leben gezeichnet. Ihre Gottlosigkeit gilt dem Verfasser als ein Zeichen der Zeit, eben der letzten Zeit, in der die Bosheit nach apokalyptischem Glauben ihren Höhepunkt erreicht.

Aber wie die Bosheit, so erreicht nun auch die Bedrängnis der Frommen ihren. Höhepunkt. Das beschreiben die c. 8-9. Wieder streiten sich die Ausleger, ob Zukunft oder Erlebtes beschrieben werde. Manche finden auch hier eine aus reiner Phantasie entsprungene Schilderung der letzten Heimsuchung Israels. Aber auch hier spricht die lebendige und konkrete Art der Angaben, die durchaus keine typischen Züge der Eschatologie bieten, dafür, daß wirklich Erlebtes geschildert wird. Mit Recht sagt schon Colani: tous les traits sont d'une extrême précision. Es ist bemerkenswert, daß auch Forscher wie Charles und Schürer, trotz sonst entgegenstehender Beurteilung des Zusammenhangs, sich diesem Eindruck nicht entziehen können. Aber wie bedenklich sind ihre Versuche, sich den Schwierigkeiten zu entziehen! Beide meinen, daß c. 8-9 die Religionsverfolgung unter Antiochos Epiphanes schildern. Nur Charles zieht mutig die Folgerung und stellt c. 8-9 geradeswegs hinter c. 5. Aber dieser kritische Gewaltstreich verschlimmert statt $\mathrm{zu}$ bessern. Die Verfolgung von c. 8-9 stellt offensichtlich die höchste Steigerung der Not dar, kann also keinesfalls vor dem geringeren Leiden von c. 6 ertragen werden, sondern muß unmittelbar vor c. 10, dem Eintritt des Endes, stehen. Es kommt hinzu, wie schon Burkitt gegen Charles bemerkt, daß die Gotteserscheinung in c. 10 sich aufs beste an die Schilderung der leidenden Frommen in c. 9 anschließt, während sie äußerst schlecht auf die Beschreibung der Gottlosen in c. 7 folgen würde. Burkitt selbst, der c. 8-9 als reines Zukunftsbild auffaßt, glaubt, der Verfasser habe das Bild der letzten Wehen nach den Erzählungen über die Märtyrer unter Antiochos Epiphanes aufgefüllt. Das nähert sich der Meinung Schürers (III 216 Anm. 46), welcher annimmt, der Verfasser lasse in c. 8 den Moses prophezeien, daß in der Endzeit Zustände eintreten sollten, wie sie außerdem nur einmal vorher, nämlich in der Zeit des Antiochos Epiphanes, kommen würden. Der ganze Zusammenhang von c. 8-9 soll nach ihm nur eine zum Vergleich herangezogene Schilderung der Verfolgungszeit unter Antiochos sein, wobei in c. 9 eine ähnliche Sage wie 2 Makk 7 vorliege. Schürer ist freilich selbst nicht recht zufrieden mit dieser gekünstelten Auffassung; er findet es mit Recht auffällig, 
daß eine so ausführliche Schilderung der Antiochoszeit nicht vorher, sondern erst in diesem Zusammenhange gegeben werde, und beruhigt sich am Ende bei dem ganz ungerechtfertigten Verdacht, daB der Text in Verwirrung geraten sei, ohne doch den von Charles betretenen Weg zu gehen.

Eine Deutung der c. 8-9. auf die Zeit des Antiochos Epiphanes ist nicht nur haltlos; - denn was Charles an übereinstimmenden Einzelheiten anführt, sind ganz allgemeine Dinge, die auf jede ähnliche Verfolgung zutreffen würden, - sondern völlig ausgeschlossen. Das beweist vor allem der Name des in c. 9 auftretenden TAXO. Es ist ganz unerfindlich, warum der Verfasser, wenn er eine Person längst vergangener Tage - etwa mit Burkitt und Charles den Eleazar von 2 Makk 7 - beschreiben wollte, deren Namen durch ein derartiges Rätselwort verhüllte. Sinn hatte eine solche Geheimniskrämerei nur dann, wenn TAXO eine Persönlichkeit der Gegenwart war, deren Name dem Uneingeweihten nicht verraten werden sollte.

Handelt es sich demnach in c. 7-9 um geschichtliche Ereignisse, die hinter den Tod des Herodes $(6,7)$ fallen, so bestätigt sich auch von hier aus die Beziehung der Stelle 6,8-9 auf den Krieg des Titus. C. 8 dagegen kann auf die Zeit des Titus nicht bezogen werden; denn Titus hat die Religion der Juden nicht verfolgt. Nur die hadrianische Religionsverfolgung kann in c. 8 gemeint sein. Da aber der Krieg des Titus unmöglich verschwiegen werden konnte, so muß er notwendig in 6, 8-9 gemeint sein.

Erst jetzt versteht man die Stelle 8,1, welche mit Merx und Charles auf Grund von 9,2 wiederherzustellen ist: Et [al]te[ra] veniet in eos ultio et ira, quae talis non fuit in illis a saeculo usque ad illud tempus, in quo suscitabit illis regem regum terrae et potenta[n]tem a potentia magna. Die erste Heimsuchung, der diese zweite gegenübergestellt wird, kann nicht die Eroberung Jerusalems durch Nebukadnezar und das babylonische Exil sein, sondern nur die Eroberung der Stadt durch Titus. Daß Israel in der Spanne eines einzigen Menschenlebens zwei so furchtbare Katastrophen hat durchmachen müssen, eben das ist das Beispiellose, was der Verfasser empfindet und was auch TAXO hervorhebt, wenn er 9,2-3 zu seinen Söhnen spricht: Videte, filii, ecce ultio facta est in plebe altera crudelis immunda et traductio sine misericordia et eminens principatum. ${ }^{1}$ Quae enim gens aut quae regio

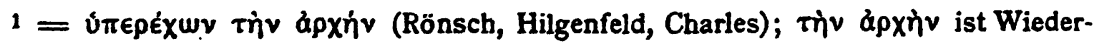
gabe des Hebräischen השונה 
aut quis populus impiorum in Dominum, qui multa scelesta fecerunt, tanta mala passi sunt, quanta nobis contegerunt! d. h.: „Sehet, meine Söhne, jetzt ist über das Volk hereingebrochen eine zweite grausame (und) unreine Heimsuchung und eine erbarmungslose Bestrafung, welche die erste übertrifft. Denn welches Geschlecht oder welches Land oder welches Volk derer, die gottlos waren gegen den Herrn, die viele Frevel begingen, hätten je so große Leiden erdulden müssen, wie sie uns betroffen haben!"

Die hadrianische Judenverfolgung ist die schwerste, die die Juden im Altertum durchgemacht haben. Sie war grundsätzlich eine Religionsverfolgung, wie dies Spartian (Hadr. 14) bezeugt: moverunt ea tempestate et Judaei bellum, quod vetabantur mutilare genitalia, - ein Verbot, das bis auf Antoninus Pius bestanden hat (vgl. Modestinus, Dig. XLVIII 8,11 pr.). Eben dies Beschneidungsverbot stellt auch unser Apokalyptiker an die Spitze seiner Beschreibung, wenn er Hadrian, den „König der Könige der Erde“, befehlen läßt, „diejenigen, die die Beschneidung bekennen, ans Kreuz zu hängen, die aber, die sie verleugnen (l. negantes), zu foltern und gefesselt ins Gefängnis zu führen, und ihre Frauen unter die Heiden zu verteilen, und ihre männlichen Kinder von Ärzten operieren zu lassen, um ihnen eine Vorhaut darüber $z u$ ziehen $(8,1-3)$." Des weiteren beschreibt er, wie die Frommen durch Martern, Feuer und Schwert gezwungen werden, unreine Götzenbilder öffentlich zu tragen, heidnische Tempel (?) zu betreten und das Wort (= Gott), die Gesetze und das Opfer (?) zu lästern $(8,4$ u. 5). Hier ist die Deutung der Einzelheiten zum Teil unsicher, aber das Wesentliche ist klar: die gewaltsame Nötigung der Frommen zu allerlei heidnischen Handlungen. . Unsere dürftigen nichtjüdischen Nachrichten über die hadrianische Verfolgungszeit erzählen hiervon nichts; aber die jüdische Überlieferung berichtet einstimmig, daß die damalige Religionsverfolgung über das bloße Beschneidungsverbot hinausgegangen sei. So erzählt Juda ben El'ai, ein Zeitgenosse, von mancherlei Behinderungen der Religionsübung "in der Stunde der Bedrängnis" (בשעת הדחק (בשעת הסכנה) vgl. tos. Sukka 1 Z. 192, 19; 2 Z. 195, 5; Erub. 8 Z. 147, 26 ed. Zuckerm. Es ist nicht nötig, diese jüdischen Nachrichten grundsätzlich anzuzweifeln; möglicherweise waren solche Bedrängnisse nicht von staatswegen angeordnet, sondern Auswüchse pöbelhaften Hasses, wie sie bei so manchen Judenhetzen in den hellenistischen Städten vorgekommen sind.

Ist c. 8 eine Beschreibung der Verfolgung unter Hadrian, so gehört auch der rätselhafte TAXO des neunten Kapitels in diese Zeit. 
Daß er eine Gestalt der Gegenwart, nicht der Vergangenheit oder der Zukunft ist, dafür bürgt, wie schon gesagt, die geheimnisvolle Verhüllung seines Namens. Auch die konkrete Angabe, die ihn „einen Mann vom Stamme Levi“ nennt, spricht dafür, daß er kein bloßes Zeugnis apokalyptischer Phantasie ist. Er ist also nicht etwa der erwartete Messias aus Juda, sondern ein Priester. Auch seine sieben Söhne und die Flucht in die Höhle werden geschichtlich sein. $\mathrm{Zu}$ allen Zeiten waren die Höhlen Palästinas die Zuflucht der Verfolgten; auch aus der hadrianischen Zeit wird das berichtet, sowohl bei Cassius Dio $(69,12$ f.), als in der talmudischen Überlieferung (b. Schabb. 33b, vgl. jer. Scheb. 29a), nach welcher Simeon ben Jochai sich mit seinem Sohne Eleazar zwölf Jahre lang in einer Höhle verborgen haben soll. Ich kann mich nicht davon überzeugen, daß diese Flucht in die Höhle nur aus dem mythologischen Motive der Siebenschläferlegende zu erklären sei, wie dies zuletzt z. B. M. Huber (Die Wanderlegende von den Siebenschläfern 1910, S. 405 ff.; 450) wieder vertreten hat. Sind denn etwa alle Erzählungen vom Fliehen in die Höhlen Palästinas, von dem selbst die archäologische Forschung so beredt zu melden weiß, nichts als mythologische Legende? Gerade das Kennzeichnende der Siebenschläferlegende, eben das Hinwegschlafen über die Zeit der Not, fehlt in unserer Apokalypse, welche ihren TAXO 9, 6-7 erklären läßt: „Wir wollen drei Tage lang fasten und am vierten Tage in eine Höhle auf dem Felde gehen und lieber sterben, als die Gebote des Herrn der Herren, des Gottes unserer Väter, übertreten; denn wenn wir das tun und sterben, wird unser Blut vor dem Herrn gerecht werden." Dieser TAXO erwartet also keinen rettenden Schlaf, sondern den Tod des Märtyrers.

Zahlreich sind die Versuche, den Rätselnamen TAXO zu deuten. Ich glaube, daß sich die Frage erledigt hat, seit Burkitt (Hastings Dictionary of the Bible III 449) seine einleuchtende Lösung gegeben hat. Er erklärt TAXO als Verstümmlung von TAXOC ${ }^{1}=\tau \alpha \xi w k$, und dieses als Wiedergabe eines hebräischen תוסת, ${ }^{2}$ was sich nach der Methode אלעור. אברב in auflöst. Burkitt deutet diesen Eleazar auf den

1 Ubrigens steht im Palimpseste hinter TAXO ein Pünikt, der möglicherweise auf den Ausfall eines Buchstabens hinweisen könnte.

2 Dem griechischen $\xi$ entspricht in hebräischer Wiedergabe regelmäBig die Buch-

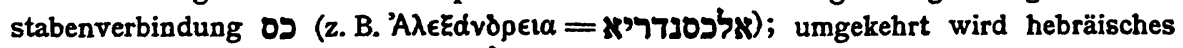

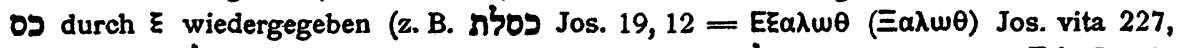

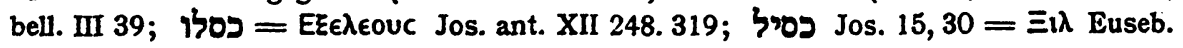
onom. 285, 46 ed. Lag.). 
frommen Schriftgelehrten von 2 Makk 6, $18 \mathrm{ff}$;; 4 Makk 5, 3, die sieben Söhne auf die sieben Söhne der Witwe von 2 Makk 7 und vergleicht zur Flucht in die Höhle 1 Makk 1,53; 2, 31. Meine Bedenken gegen diese Beziehungen auf die Zeit des Antiochos habe ich schon vorgebracht. Welch eine heillose Verwirrung von Überlieferungselementen muß Burkitt annehmen! Was interessiert den Apokalyptiker überhaupt jener obskure Eleazar einer längst vergangenen Zeit!

Eleazar ist. ein Zeitgenosse des Apokalyptikers, ein angesehener Frommer der hadrianischen Zeit, ein Mann priesterlichen Geschlechtes, der sich mit seinen sieben Söhnen vor den Verfolgern in einer Höhle verbirgt, um das Gesetz nicht übertreten zu müssen. $O b$ eine uns auch sonst bekannte geschichtliche Persönlichkeit gemeint ist, läßt sich natürlich nicht beweisen. Es könnte naheliegen, ihn jenem „Priester Eleazar" gleichzusetzen, den uns die Münzen der Barkochbazeit (Madden, Coins of the Jews 1881) kennen gelehrt haben. Einer der Münztypen dieser Zeit trägt bekanntlich die Aufschrift: אלצור הכוהן und die Gegenמנת אחת לגאלת ישראל (Madden p. 198), und diesem Typus entsprechen die gleichzeitigen Münzen Simeons mit gleicher Gegenschrift und mit den Aufschriften: שמעון נשיא ישראל (Madden p. $203 \mathrm{ff}$.) bzw. ירושלם (Madden p. 202). Das Münzzeugnis ergibt also, daß im Jahre 132 n. Chr. neben dem Fürsten Simeon und der Stadt Jerusalem auch ein Priester Eleazar Münzen geprägt hat. Ein solcher münzenprägender Priester, der sich außerdem als "der Priester" schlechthin bezeichnet, kann aber kaum ein anderer als der Hohepriester jener Zeit gewesen sein. הכוהן ist alter und vornehmer Ausdruck für den Hohenpriester (vgl. Ps 110,4; Sir 50, 1 hebr.). Daß ein solcher nach der Befreiung Jerusalems wieder eingesetzt worden ist, erscheint ganz natürlich. In der rabbinischen Überlieferung werden aus der Zeit Hadrians verschiedene angesehene Träger des Namens Eleazar genannt: Rabbi Eleazar (oder Eliezer) von Modeim, der Oheim Barkochbas, Rabbi Eleazar ben Azarja und Rabbi Eleazar ben Charsom. Die beiden letzteren waren Priester. $\mathrm{Ob}$ der eine oder der andere mit dem „Priester Eleazar“ der Münzen gleichzusetzen ist, läßt sich nicht ausmachen. ${ }^{1}$

Aus dem "zweiten Jahre der Freiheit Israels“ besitzen wir keine Eleazar-Münzen. Man könnte versucht sein, anzunehmen, daß Eleazar damals nicht mehr Hoherpriester gewesen, vielleicht als Märtyrer gestorben war; doch kann das Fehlen von Münzen auch Zufall sein.

1 An den ersteren denkt Hamburger (Zeitschrift für Numismatik XVIII 1892 S.311ff.), an den letzteren Schlatter (Die Tage Trajans und Hadrians S. $54 \mathrm{ff}$.). 
Die Flucht des TAXO = Eleazer und seiner Șöhne in die Höhle bezeichnet für den Apokalyptiker den Höhepunkt der Not. In der Erwartung des nahen Gottesreiches wollen sie lieber Hungers sterben, als das Gesetz übertreten und ihr Judentum verleugnen. Es leuchtet ein, daß wir uns hier in Kreise derjenigen Frommen befinden, aus deren Mitte unsere Apokalypse hervorgegangen ist. Jetzt - das will der Verfasser tröstend seinen Leidensgenossen sagen - jetzt steht die Erscheinung des rettenden Gottes unmittelbar bevor. Wer bis an das Ende beharrt, der wird selig. Zu dichterischer Form steigert sich hier, wo sie wahrhaft weissagend wird, - ähnlich wie in der Schrift der Testamente, - die erregte Rede (c. 10); denn jetzt soll ausgesprochen werden, worauf alle mit zitternder Spannung warten:

Dann erscheint seine Herrschaft über all seine Schöpfung,

Dann hat der Satan ein Ende,

Und die Trauer schwindet mit ihm dahin.

Dann' erhält Auftrag der Engel,

Der an der Spitze steht,

Und rächt sie alsbald an ihren Feinden.

Denn der Himmlische steht auf vom Thron seiner Herrschaft

Und zieht aus von seiner heiligen Wohnung

In Grimm und Zorn um seiner Kinder willen.

Und die Erde erbebt, zittert bis zu ihren Enden,

Und die hohen Berge werden erniedrigt,

Und die Hügel zittern und fallen.1

Die Sonne gibt keinen Schein und wandelt sich in Dunkel,

Des Mondes Hörner zerbrechen, und er wandelt sich ganz in Blut,

Und der Sterne Krejs verwirrt sich.

Und das Meer entweicht bis in den Abgrund,

Und die Quellen der Wasser versiegen,

Und die Flüsse trocknen aus.2

Denn aufsteht der Höchste, der ewige Gott allein,

Und erscheint, um die Heiden zu strafen,

Und vernichtet all ihre Götzenbilder.

Dann wirst du selig werden, Israel,

Wirst steigen auf Adlers Nacken und Fittiche,

Und zu Ende werden sein (die Tage deiner Leiden).

1 Der Text ist verderbt: et concutientur et convalles cadent.

. 2 lies exarescent. 
Und Gott wird dich emporheben,

Und dich wohnen lassen am Himmel der Gestirne, 1

....... am Ort ihrer Wohnstatt.

Und aus der Höhe wirst du schauen, und deine Feinde in der Hölle sehn. ${ }^{2}$ Du wirst sie erkennen und dich freuen,

Wirst danksagen und deinen Schöpfer lobpreisen.

Scharf trennt der Apokalyptiker die zwei Weltzeiten: jetzt Satansherrschaft, einst Gottesherrschaft, jetzt Trauer, einst Seligkeit, Freude, Dank und Lobpreis. Jetzt herrschen Israels Feinde, die Heiden mit ihren Götzenbildern, zu deren Dienst man auch "die Kinder Gottes" zwingen möchte. Aber der oberste der Engel Gottes, Michael, Israels Schutzherr (vgl. Ap. Joh. 12, 7ff.), wird in des Höchsten Auftrage eingreifen und Israel an seinen Feinden rächen. Dann hat der Satan ein Ende, die Heiden werden gestraft, ihre Götzenbilder vernichtet. Israel aber wird zur Seligkeit der Kinder Gottes in die Höhen des Sternenhimmels entrückt, von wo es die Verdammnis seiner Feinde in der Hölle schauen darf (vgl. dazu Ev. Lc 16, 23ff.).

2.

Nur aus einer Zeit schwerster Leiden begreift sich diese Apokalypse leidenschaftlichsten Hasses gegen Rom. Es ist bemerkenswert, daß wir ähnliche Drohungen gegen Rom nur aus der Zeit nach 70 kennen, nämlich IV Esr 6, 8-10; 11-12; Ap Bar 11.39, 5-7; Ap Joh 13. 17, vgl. auch Jos. ant. X 276. Als unser Apokalyptiker schrieb, stand die Existenz Israels wirklich auf dem Spiele. Man lese 12, 10-12: "Darum werden die, die Gottes Gebote halten und erfüllen, wachsen und glücklich leben; denen aber, die sündigen und die Gebote außer acht lassen, werden die verheißenen Güter mangeln, und sie werden von den Heiden mit vielen Martern gestraft werden; daß er sie aber ganz und gar ausrotte und verlasse, ist unmöglich!“ Es leuchtet ein, daß die Judenschaft zur Zeit des Varus vor einer solchen Möglichkeit nicht stand, wohl dagegen nach 70 (vgl. dazu IV Esr 5, 28; Ap Bar 3, 5. $9 ; 48,19 ; 78,7$; gr. Ap Bar 1). So konnte man nur schreiben in einer Zeit, da alles in. Frage gestellt war. Es ist die Zeit, in der der Seher der Esraapokalypse seine bangen Seufzer laut werden läßt.

1 Et faciet te herere caelo stellarum.

2 Der überlieferte Text: et vides inimicos tuos in terram ist kaum haltbar. Charles setzt griechisches $\epsilon^{2} \nu$ rh voraus, was er aus zugrunde liegendem hebräischem בגיא הנם erklärt. $Z u$ der hier vorausgesetzten Transskription des hebräischen Aus-

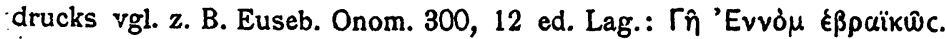


Auch unser Apokalyptiker klammert sich mit heißem/ Glauben an die Zusagen Gottes, und immer wieder weist er darauf hin, daß Gott dem Volke das Land zum Eigentum gegeben, ihm dessen Besitz durch Bund und Schwur verheißen habe $(1,8 \mathrm{f} . ; 2,1.7 ; 3,9 ; 4,2.5 ; 11,11.17$; 12,13). Israel ist ja das auserwählte Volk $(4,2)$, der Erstling der Kreatur $(1,13)$. Um Israels willen - so tröstet sich der Verfasser $(1,12)$ ähnlich wie andere Schriftsteller der Zeit nach 70 (IV Esr 6,55.59; 7, 11; Ap Bar 14;19; 15, 7; 21, 24, vgl. Exod. r. 28) - ist ja die Welt geschaffen worden. ${ }^{1}$ Jetzt, wo alle äußeren Stützen zerbrochen scheinen, stützt man sich auf den Glauben an die Fürbitte Moses, des großen Mittlers $(1,14 ; 3,12),{ }^{2}$ der nicht nur während seines irdischen Lebens, sondern auch jetzt noch nach dem Tode in himmlischer Glorie für das Volk Fürsprache einlegt $(11,17 ; 12,6)$, ähnlich, wie es sonst nach zeitgenössischer Vorstellung Jeremia tut (2 Makk 15, 14; Ap Bar 2, 2; Paral. Jer 2,3). In diese Stimmung gehört auch die herzbewegende Klage Josuas über den Hingang Moses (11,4ff.), die ihre Parallelen IV Esr 12, $41 \mathrm{ff}$. in der Klage des Volkes über Esras bzw. Salathiels Scheiden, oder Ap Bar 32, 9ff. (vgl. 77, 12) in der ähnlichen Klage über den Hingang Baruchs hat. ${ }^{3}$

Kennzeichnend für die Stimmung der Frommen nach 70, als der judäische Staat untergegangen war, ist die Anteilnahme am Schicksal ganz Israels, d. h. auch der zehn Stämme. Aus der Mischna erfahren wir, wie Rabbi Akiba und Rabbi Eliezer über die Rückkehr der zehn Stämme sich stritten; jener leugnete, dieser erwartete sie (m. Sanh. $X$ 3). Auch die zeitgenössische Apokalyptik beschäftigt sich mehrfach mit der Frage. Wo sind die zehn Stämme hingeraten? Der Bibeltext sagt (Dt. 29, 27): „in ein anderes Land“ (אריץ אחרת). Das wiederholt unser Verfasser $(4,3)$. Man weiß auch, wo dies "andere Land“ zu suchen ist. Josephus (ant. XI 133) erklärt, daß nur zwei Stämme in Asien und Europa den Römern untertan seien, während die übrigen zehn als zahllose Menge jenseits des Eufrats wohnten. IV Esr 13, 39ff.

1 In christlicher Umdeutung Herm. vis. II 4,1 vgl. vis. I 1, 6 IV, V. Daneben findet sich sonst der Gedanke, daB die Welt um des Menschen willen geschaffen sei (IV Esr 8, 1.44; Ap Bar 14,18; Herm. mand. XII 4 und oft in der nachapostolischen Literatur).

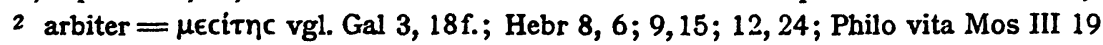
und häufig im Talmud (7)סר) vgl. Levy, Lex. III $595 \mathrm{f}$.

3 Auch der Ausdruck "scheiden" (discedere) im Sinne von "sterben" $(11,4.9)$ begegnet Ap Bar 14,19; 15,1; 43, 2 und sonst wieder. Ferner ist IV Esr 14, 19 zu vergleichen, wo Esra zu Gott spricht: „Ich scheide jetzt, wie du mir befohlen hast, und will das Volk, das jetzt lobt, (noch einmal) unterweisen. Aber die später Geborenen, wer wird sié belehren? ${ }^{\alpha}$ 
führt diese Wanderung über den Eufrat in das "andere Land“ (arzareth =ארץ אחרת) legendenhaft aus, und Baruch (Ap'Bar 77f.) schickt durch einen Vogel ein Sendschreiben an die neuneinhalb Stämme jenseits des Eufrats. Ap Joh 7, 4-8 erwartet die Versiegelung der 144000 aus allen zwölf Stämmen. All das sind Zeugnisse gerade der Zeit nach 70 für die Hoffnung auf eine Wiederherstellung des ganzen Zwölfstämmevolkes. Deshalb betont auch unser Apokalyptiker überall die Solidarität der zwölf Stämme: beide Teile des Volkes haben gesündigt $(3,5)$, beiden gemeinsam gilt die Verheißung $(3,9)$; Israels wegen - nicht nur wegen Judas und Benjamins - wird Gott einst einschreiten und Israel zum Himmel emporheben $(10,8)$. Das ist der gleiche Gedanke, für den auch Ap Bar 78, 4 sich erwärmt: alle zwölf Stämme sind in éine Gefangenschaft geführt worden; ärger als der zehn Stämme waren die Sünden der zwei Stämme (Ap Bar 1,2); nun hat auch sie, wie jene, das Unglück betroffen (Ap Bar 79,3). Gerecht war die Strafe; das möge man erkennen und Buße tun $(3,5, \operatorname{vgl}$. Ap Bar 78,5); denn am "Tage der Buße" $(1,18)$, der großen Buße des ganzen Volkes, wird das Reich Gottes erscheinen (vgl. b. Sanh. 97 b; Pirke de R. Eliezer 43; Pesikta 163b).

Vergegenwärtigen wir uns die Lage der palästinischen Juden nach 70. Die nationale Behörde zu Jerusalem, das Hohepriestertum und das Synedrion waren verschwunden. Die innerjüdische Administration und Rechtsprechung lag fortan ganz bei den einzelnen lokalen Gerichtshöfen. ${ }^{1}$ Die Rolle, die diese in der Zeit unsers Apokalyptikers spielten, spiegelt sich in 2,2; der Verfasser hält es für nötig, die Einsetzung dieser lokalen Behörden nach Moses Befehl an Josua ausdrücklich zu betonen. Sie erscheinen als die legitime jüdische Obrigkeit. Die Herrschaft haben freilich zurzeit andere, die aufs schärfste angegriffen werden (7, 3-9). Wen meint der Verfasser mit ihnen? Man hat die verschiedensten Vorschläge gemacht: Pharisäer, Sadduzäer, Herodeer, römische Prokuratoren, die Rabbinen nach 70 . Unmißverständlich kennzeichnet sie der Satz 7, 3: „und es werden über sie herrschen verderbliche und gottlose Menschen, welche sagen, sie seien fromm" (lies: et regnabunt de his homines pestilentiosi et impii dicentes se esse justos). Der Satz sagt deutlich, daß die betreffenden einerseits über die Juden herrschen werden, andererseits sich selbst als Juden angesehen wissen wollen, wie dies auch ihre Beobachtung der Reinheitsvorschriften

1 Vgl. Chajes, Les juges juives en Palestine de l'an 70 à l'an 500 (Revue des études juives t. XXXIX 1899 p. 39-52). 
$(7,10)$ bestätigt. Daraus ergibt sich, daß weder Pharisäer noch römische Prokuratoren gemeint sein können. Auch auf die sog. Sadduzäer paßt der Satz schwerlich, selbst wenn man in herkömmlicher Weise Hohenpriesteradel und Sadduzäertum gleichsetzt; denn von einer "Herrschaft" des Hohenpriesteradels oder der Sadduzäer (vgl. dazu z. B. Jos. ant. XVIII 17) über die Juden konnte weder zur Zeit der Herodessöhne noch unter den römischen Prokuratoren gesprochen werden. Die Deutung wird vollends unmöglich, wenn die Gottlosen von 7,3-9 erst der Zeit nach 70 angehören, wo für das sog. "Sadduzäertum" sowieso kein Raum mehr ist. Auch die Deutung auf das Rabbinentum der späteren "Zeit, auf die Synedrien von Jabne und Uscha paßt nicht recht; denn was Colani als Beispiele der fürstlichen Pracht des Patriarchats anführt, bezieht sich erst auf nachhadrianische Zeit, vor allem auf die Blütezeit des Patriarchats vor Tiberias seit Juda I. Am ersten möchte ich in jenen Gottlosen Leute wie die jüngeren Herodeer sehen, z. B. Männer wie Agrippa II., Herodes von Chalkis und ihren romanisierten Anhang. Mit unzweideutigen Worten geißelt der Apokalyptiker das üppige Wohlleben, das fürstliche Gepränge und das Scheinjudentum dieser Römlinge. Man begreift es, daß er, der glühende Hasser Roms, gerade in der Römerfreundschaft dieser Juden den Gipfel des Verderbens erblickte.

Wenn unsere Apokalypse ein Zeugnis der sonst an Geschichtsurkunden für uns so armen hadrianischen Zeit ist, so ist es für ihr Verständnis besonders wichtig, sich die damaligen Zustände in Jerusalem und Judäa deutlich klarzumachen. Schürer (I 649) glaubte, aus Jos. bell. VII 3 herauslesen zu dürfen, daß Jerusalem so gründlich dem Erdboden gleichgemacht gewesen sei, „daß es den Besuchern nicht einmal den Glauben ermöglichte, es sei je bewohnt gewesen“. Aber was Josephus hier schreibt, ist, wie so oft bei ihm, Rhetorik, und bezieht sich außerdem nicht auf die Stadt, sondern nur auf den größeren Teil der Stadtmauern. Jerusalem war seit 70 die Garnisonstadt der zehnten Legion, die den alten herodianischen Palast als Kaserne benutzte. Dieser Palast mit seinen starken Türmen war, wie auch Josephus erzählt, nicht zerstört worden. Auch sonst war die Stadt nicht vom Erdboden verschwunden. Hadrian, heißt es bei Appian (Syr. 50), zer--

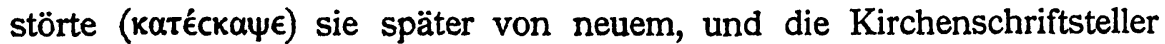
wiederholen das mehrfach. Eusebius (dem. ev. VI 18,10 ed. Gaisf.) er-

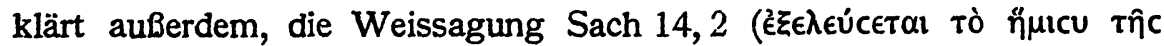

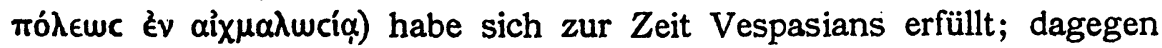
die andere Hälfte der Stadt, d. h. der Einwohnerschaft, sei zur Zeit 
Hadrians belagert und vertrieben worden. Jerusalem hatte also auch nach 70 eine, wenn auch stark verminderte, jüdische Bevölkerung. Das will auch die rabbinische Überlieferung wissen. Aus tos. Jom tob 3 Z. 205, 26f. erfahren wir, daß z. B. Rabbi Eleazar ben Rabbi Sadok und Abba Schaul ben Batnith in dieser Zeit in Jerusalem wohnten

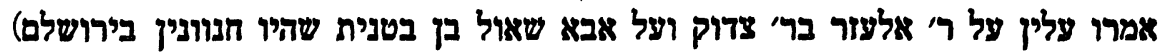
כל ימיהם (כלה (כ). Die sieben Synagogen, von denen der Pilger von Bordeaux und Epiphanius (de mens. et pond.14) erzählen, daß nur noch eine von ihnen übriggeblieben sei, stellen vielleicht die Zahl der nach 70 erhaltenen Synagogen Jerusalems dar. Auch die Apokalypse Baruchs spiegelt wohl Zustände der eigenen Zeit wieder, wenn sie "Volk“ beim Tempel wohnen läßt.

Wie stand es mit dem Tempel selbst? Titus hatte, wie Josephus behauptet, nicht die Absicht gehabt, den prächtigen Tempel der Juden zu zerstören; nur durch die Unvorsichtigkeit eines Soldaten war das Tempelgebäude, der היכל, am 17. Tammuz ein Raub der Flammen geworden. Seitdem hatte das tägliche Opfer aufgehört (Jos. bell. VI 94; $\mathrm{m}$. Taanith IV 6). Daß die Römer alle Baulichkeiten des heiligen Platzes dem Erdboden gleichgemacht hätten, ist weder bezeugt noch wahrscheinlich. Um von der Tempelburg Antonia zu schweigen - Befestigungen solcher Art zu zerstören, war nicht römische Art -, so sind jedenfalls noch heute Reste des herodianischen Tempels, eingebaut in die Substruktionsbauten des heutigen Harams, erhalten, so die im Stil eigenartigen Säulen des Doppeltores im Süden (vgl. dazu H. Thiersch und G. Hölscher, in Mitteilungen der Deutschen Orientgesellschaft 1904, Nr. 23 S. 37 f.). Vom Vorhandensein der für das Brennholz bestimmten Zelle berichtet die sogleich anzuführende Stelle tos. Edujoth 3 Z. 459, 25. An derselben Stelle bezeichnet Rabbi Josua den Tempel auch nach 70 noch immer als "unser Haus“.

Auf jeden Fall benutzten die Juden auch nach 70 den Tempelplatz als Betplatz. Josephus läßt den Eleazar von Masada im Jahre 73 sprechen: „Elende Greise sitzen bei der Asche des Heiligtums und wenige Weiber, die zu schamloser Lust von den Feinden aufbewahrt sind" (bell.VII377). In der Apokalypse Baruchs sehen wir 'Baruch vor den Toren des Tempels sitzen $(10,5)$ und auf den Tempelplatz gehen $(13,1 ; 21,2 ; 25,1)$. Weiteres erzählen rabbinische Nachrichten (vgl. Schlatter, Die Tage Trajans und Hadrians 1897). Wir hören von einem Streitgespräch des Rabbi Josua über die Frage, ob der Tempelhof für unrein erklärt werden dürfe, weil Knochen in der für das Brennholz bestimmten Zelle gefunden wurden (tos. Eduj. 3 


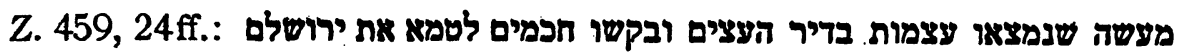

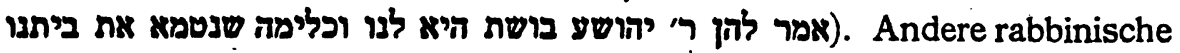
Nachrichten erzählen, wie Rabban Gamliel II. auf dem Tempelhofe um-

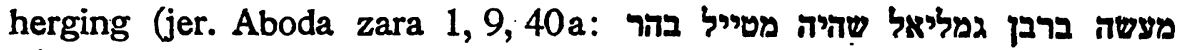
הבית וראה אשת אחת גויה וברך עליה (החור), wie Gamliel, Eleazar ben Azarja, Josua und Akiba nach Jerusalem wallfahrteten (Sifre, Ekeb 43; Midr. r. zu Thren 5,18), wie Simeon ben Jochai nach Jerusalem pilgerte (tos. Meila 1 Z.557, 8: אמר ר' שמעון כששבתי בכפר בית פאגי (אלית), wie eine Frau zur Zeit des Josua ben Chalafta nach Jerusalem wallfahrtete (tos. Ned. 5

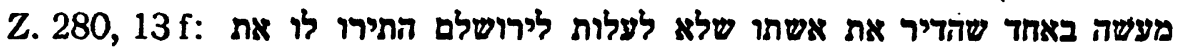
נדרו ועלתה), wie Ben Zoma Volkshaufen auf dem Tempelplatze sah (tos. Ber.7 Z.14, 24: בן זומא ראה אוכלסין בהר הבית). Alle diese Zeugnisse stimmen darin überein, daß der Tempelplatz auch nach 70 noch eine Betstätte der Judenschaft gewesen ist.

Man hat in den Kreisen der Gelehrten sogar ernstlich die Frage erwogen, ob nicht trotz der Zerstörung des Tempelhauses und der Mauern der Opferkult stattfinden dürfe. In einem Ausspruche des Rabbi Josua, der nicht wie eine bloß schulmäßige Erörterung klingt, wird diese Frage mit ja beantwortet:

Ich habe gehört, daß man Opfer darbringen darf, auch wenn kein Tempel da ist;

daß man Hochheiliges essen darf, auch wenn keine Scheidewand (um den Vorhof) da ist;

daB man Heiliges geringeren Grades und zweiten Zehnt essen darf, auch wenn keine Mauer um Jerusalem ist;

denn die erste Einweihung hat geheiligt sowohl für ihre als für die künftige Zeit.

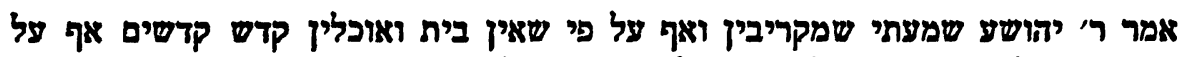

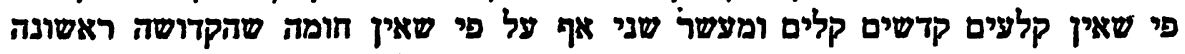
Edujoth VIII 6; vgl. dazu noch tos. 3

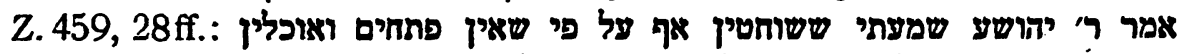

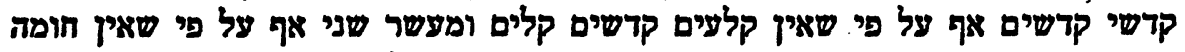

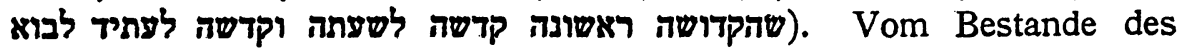
Tempelhauses war der gewöhnliche Opferkult in der Tat unabhängig; denn er fand statt im Tempelhofe auf dem Brandopferaltar, jener gewachsenen Felskuppe des Zionsgipfels, die unzerstörbar die Zeiten überdauernd noch heute das Heiligtum der Kubbet eş-șachrā bildet. Wie man einst nach der Zerstörung des Tempels durch Nebukadnezar hier auch durch das Exil hindurch geopfert hatte, so wäre auch nach 70 hier die Möglichkeit zum Opfern vorhanden gewesen. Ob man es wirklich getan hat, muß freilich bezweifelt werden. Auch unsere 
Apokalypse scheint dies nicht vorauszusetzen; denn vielleicht spiegeln sich Zustände der Zeit nach 70 auch in den Worten 4, 8, welche die Lage der Judäer nach dem Exil beschreiben: sie trauern und seufzen, weil sie dem Herrn ihrer Väter keine Opfer darbringen können (tristes et gementes quia non poterint referre immolationes domino patrum suorum, vgl. dazu Ap Bar 68, 6; auch Hen 89, 73).

Hält man sich diese Zustände nach 70 vor Augen, so wird man schwerlich Anstọ nehmen an der Stelle 1,17 f., wo Jerusalem als der Ort bezeichnet wird, den Gott „von Anfang der Weltschöpfung an machte, auf daß sein Name daselbst angerufen werde bis zum Tage der Buße, bei der Heimsuchung, mit der der Herr sie heimsuchen wird am Ende der Tage". Auch nach 70 hat man, wie dargelegt, an der Stätte des Heiligtums den Namen Gottes angerufen, ja damals mit größerer Inbrunst und Bußfertigkeit als je. Inniger als je wird man sich jetzt festgeklammert haben an dem Glauben, daß der Tempel die Stätte der Anrufung bleiben solle bis zum Ende der Tage, „bis zum Tage der Buße“. Ja, vielleicht verstehen wir jetzt auch die Wendung 6, 9: partem aedis ipsorum. Unmöglich konnte ein gläubiger Jude, wie unser Apokalyptiker, zugestehen, daß es mit dem Heiligtum seit 70 ganz und gar aus sei. Er gibt $z u$, daß Titus einen Teil des Heiligtums mit Feuer verbrannt habe, aber daß alles verloren, der Tempel Israels für alle Zeit vernichtet sei, kann er unmöglich einräumen. Mochten die schwarzgebrannten Ruinen des Tempelgebäudes von dem furchtbaren Schicksal des Jahres 70 erzählen, der Platz des Heiligtums mit dem Altar und mancherlei Baulichkeiten, war immer noch im Besitz der frommen Gemeinde; noch immer erhoben sich an heiliger Stätte flehende Hände der Klagenden und Büßenden zum Gott der Väter, in der Hoffnung auf künftige Wiederherstellung des verwüsteten Heiligtums.

Man glaubte ja noch lange nicht daran, daß der gegenwärtige $\mathrm{Zu}-$ stand ein endgültiger sein sollte. Noch glomm das nationale Feuer heiß unter der Asche. Kaum war ein neues Geschlecht nach 70 herangewachsen, so flammten die Unruhen wieder auf. Schon in den letzten Jahren Trajans (115-117) waren in Ägypten, Kyrene und Mesopotamien Aufstände der Juden ausgebrochen, die nach Angabe des Biographen Hadrians (Spartian. Hadr. 5) auch nach Palästina überschlugen. Später erfolgte, um die Gemüter der Juden vollends aufzureizen, das Beschneidungsverbot Hadrians, - man weiß leider nicht wann. Schließlich gab Hadrian, als er $130 \mathrm{n}$. Chr. nach Syrien kam, Befehl, Jerusalem als heidnische Stadt Aelia Capitolina neuzugründen. Damit war die Erbitterung der Judenschaft zum Siedepunkt erhitzt. Als $\mathrm{Ha}$ - 
drian nach einem zweiten Aufenthalte im Jahre 131 von neuem Syrien verlassen hatte, brach - wohl im Jahre 132 - jener größte aller Judenaufstände aus, der zur zeitweiligen Befrèiung Jerusalems und zur Neugründung eines jüdischen Reiches unter dem Fürsten Simeon Barkochba führte, dem auch der Führer des Rabbinentums Akiba als dem "Gesalbten" huldigte und unter dem der Wiederaufbau des Tempels in Angriff genommen, ja auch der Opferkult vielleicht in gewissen Grenzen wieder aufgenommen wurde.

Eben diese Zeiten sind der Hintergrund unserer Apokalypse. Stärker als in anderen Apokalypsen flammt in ihr ein politisch-nationaler Geist, glühender als irgendwo ist der Haß gegen Rom, grundsätzlicher der Gegensatz des Judentums gègen das feindliche Heidentum, das als Verkörperung der satanischen Macht erscheint und gegen das Gott selbst und sein oberster Engel nunmehr am Ende der Tage kämpfen werden, um die Kinder Gottes zu rächen und Israel „auf Adlers Nacken und Fittichen" in himmlische Höhen zu entrücken.

(Schluß folgt.) 